\title{
La belleza de la expresión
}

Comunicar es un aspecto importante de la labor pedagógica que contribuye a generar comprensión, a hacer explícito el conocimiento obtenido. Aceptar el reto de la comunicación implica asumir la exigencia de la adecuada expresión que esta conlleva, pues no se trata tan solo de decir, sino de adaptar el discurso y el mensaje a nuestros interlocutores, con el imperativo de propiciar el contexto de recepción adecuado. Se trata de saber a quién queremos interpelar a propósito de qué.

La belleza de la expresión -que conjugue rigor y calidad- no es un logro fuera del alcance de nuestra condición de maestros y por tanto de laboriosos usuarios de la palabra. Como lo señala el maestro Paulo Freire, la palabra nos permite configurar la realidad del mundo, por ello, cambiar y adecuar nuestro lenguaje es una manera de cambiar el mundo. En este sentido, la palabra no es neutra, como tampoco lo es la labor del maestro, por eso tiene valor social y dimensión política y debido a ello su uso exige eticidad y reflexión.

En particular, la comunicación escrita es un oportunidad para promover la comprensión social del papel de los maestros y maestras como profesionales estudiosos de los temas de la educación, pero también como intelectuales que piensan la educación y sus relaciones con la sociedad y la vida. Constituye un medio para mostrar su faceta como sujetos políticos que reflexionan sobre el valor de la educación en el contexto de las realidades de la cotidianidad de la escuela, para trascenderla y superarla a partir de ella misma, en la búsqueda de soluciones y posibilidades a los problemas actuales de la educación colombiana.

Como miembros de la comunidad educativa, los maestros, se constituyen en legítimos interlocutores con la sociedad y, por ello, están llamados a tomar posición en defensa de su derechos y a pronunciarse frente a los discursos y anatemas que desvirtúan la valía de su trabajo. En este sentido, la escritura académica propor- ciona visibilidad a la multiplicidad de experiencias de la realidad escolar, a las relaciones que construimos de lo disciplinar con el mundo, en el compromiso con su transformación, y permite destacar las singularidades que hacen de cada experiencia, una vivencia única e irrepetible. A través de la escritura podemos expresar y conocer lo que surge de la labor y la experiencia cotidiana, pero también la reflexión que produce la escuela y la educación sobre sí mismas.

Una comunidad de educadores que tiene el propósito de comunicar sus trabajos y compartir sus innovaciones pedagógicas estimula el intercambio, fortalece vínculos académicos e inspira a otros educadores y educadoras a emular la actividad de socializar sus propias construcciones discursivas. Así, la escritura constituye una forma privilegiada de construir nuevas realidades para la actividad docente y la educación, para poner a circular nuevos sentidos, configurar nuevas realidades.

En esta medida, en la Revista Nodos y Nudos reivindicamos el poder de la palabra e invitamos a los maestros y maestras a trabajar por la comunicabilidad de las reflexiones y discursos sobre su quehacer, a propiciar la interlocución, a tender vínculos y a mostrar/conocer la realidad cotidiana de la escuela en Colombia a través del testimonio de sus protagonistas.

Abrimos nuestra páginas para documentar y evidenciar la labor realizada, reivindicar el papel del maestro y la educación como aspectos centrales en la sociedad y analizar y comprender el día a día de las relaciones que configuran el mundo escolar a través de la comunicación escrita. Y en esta medida, contribuir a fortalecer los vínculos entre los miembros de la comunidad educativa y a derribar la barrera invisible que nos separa y nos incomunica, entre nosotros y con los otros.

Juan Carlos Bustos Gómez 


\section{Rodrigo Arguello Guzmán}

Magister en Literatura Pontificia Universidad Javeriana. Filología e Idiomas, Universidad Nacional de Colombia. Actualmente es Profesor de la Universidad Tecnológica de Pereira donde dicta la cátedra Nuevos Formatos Audiovisuales. Presidente de la Asociación Latinoamericana de SIMBOANÁLISIS, sede Bogotá.

prometeusster@gmail.com

\section{Juan Carlos Bustos Gómez}

Magister en Comunicación Universidad lberoamericana, México. Comunicador Social. Pontificia Universidad Javeriana, Bogotá Docente Universidad Pedagógica Nacional. Miembro del Grupo Física y Cultura del Departamento de Física de la UPN. Investigador Colciencias y Editor serie Pre-impresos Estudiantes, UPN.

juancarlosbustos@yahoo.com

\section{Carmen Alicia Martínez Rivera}

Doctora en Educación-Didáctica de las Ciencias. Magister en Docencia de la Química. Licencia en Química de la Universidad Pedagógica Nacional. Investigadora Colciencias, Profesora en el Doctorado Interinstitucional en Educación de la Universidad Distrital Francisco José de Caldas.

camartinezr@udistrital.edu.co, carmenaliciamartinezrivera@gmail.com

Rosa Inés Pedreros Martínez

Candidata a Doctora en Educación de la Universidad Distrital Francisco José de Caldas. Maestría En Docencia de La Física Universidad Pedagógica Nacional. Licenciatura en Física Universidad Distrital Francisco José de Caldas. Docente de Planta UPN, Departamento de Física. Investigadora Colciencias grupo Corporación Escuela Pedagógica Experimental

rosapedreros@yahoo.com

\section{Olga Lucia Castiblanco Abril}

Candidata a Doctora Ensino de Ciencias e Matematicas, Universidade Estadual Paulista Júlio De Mesquita Filho. Magister en Docencia de la Física Universidad Pedagógica Nacional. Licenciatura en Física, Universidad Distrital Francisco José de Caldas. Docente universidad Distrital Francisco José de Caldas. ocastiblanco@yahoo.com

\section{Edwin German Garcia Arteaga}

Doctor en Educación, Universidad Autónoma de Barcelona. Magister en Docencia de la Física, Universidad Pedagógica Nacional. Master Europeo en Educación, Universidad Autónoma de Barcelona. Licenciatura en Física, Universidad Pedagógica Nacional. Docente Universidad del Valle e Investigador Colciencias

edwingermangarcia@hotmail.com

\section{Jairzinho Francisco Panqueva Cifuentes} Doctor en Ciencias Sociales con Especialidad en Antropología Social del Centro de Investigaciones y Estudios Superiores en Antropología Social (CIESAS), Guadalajara, Jalisco, México. Magister en Ciencias Sociales con Énfasis en Estudios Étnicos de la Facultad Latinoamericana de Ciencias Sociales (FLACSO), Ecuador. Licenciado en Educación Física de la Universidad Pedagógica Nacional. Docente Universidad Pedagógica Nacional. Docente del Colegio San Bernardino IED, Territorio Muisca de Bosa.

panqueba@gmail.com

\section{Alexander Ortiz Ocaña}

Ph. D. en Educación (Doctor en Ciencias Pedagógicas), Universidad Pedagógica de Holguín, CUBA. Doctor Honoris Causa en Iberoamérica, Consejo Iberoamericano en Honor a la Calidad Educativa. Magíster en Gestión Educativa en Iberoamérica. Magíster en Pedagogía Profesional. Experto en Afectividad, Formación por Competencias, Creatividad y Aprendizaje Basado en Problemas. Licenciado en Educación. Docente de la Universidad del Magdalena. Investigador en el Doctorado en Ciencias de la Educación de la Universidad del Magdalena en Convenio con RUDECOLOMBIA. Alexanderortiz2009@gmail.com

\section{Adriana Arribas}

Licenciada en Ciencias de la Educación de la Universidad de Buenos Aires. Docente de la carrera de Edición de la Facultad de Filosofía y Letras de la UBA. Editora Especializada en Educación.

adranaarribas@ciudad.com.ar

\section{Eva Leticia Ortiz Avalos}

Doctorante en Urbanismo por la Universidad Nacional Autónoma de México con el Tema Imaginarios Urbanos en Centros Históricos. Licenciada en Arquitectura en la UNAM. Profesora en la Licenciatura de Arquitectura. Docente en la Universidad La Salle en la Especialidad en Arquitectura de Paisaje, México. Profesora en el Instituto Tecnológico de Estudios Superiores de Monterrey campus Querétaro en la Licenciatura de Arquitectura. evaleticiaortiz@gmail.com

\section{Alfredo Manuel Ghiso Cotos}

Especialización en Desarrollo Social Análisis de Conflictos y Problemas en Programas de Desarrollo, Universidad St Fx Xavier. Maestro en Español y Literatura Universitario, Universidad Del Comahue. Especialización Instituto Paulo Freire Diploma en Lecciones de Freire. Docente Investigador, Coordinador del Laboratorio Universitario de Estudios Sociales, Grupo de Investigación Facultad de Derecho y Ciencias Políticas, Fundación Universitaria Luís Amigó. Docente a Cargo de los Cursos de Investigación Social, Sistematización de Prácticas Sociales y Pedagogía social en las Facultades de Ciencias Sociales y Humanas en la Universidad de Antioquia, Medellín, Colombia.

alfredogh@une.net.co

\section{Comité editorial}

\section{Alfredo Ayarza Bastidas}

Especialista en Gerencia Integral de Empresas, Universidad del Rosario. Miembro de la cámara Colombiana del Libro. Miembro de Funda Lectura. Bogotá, Colombia.

ayarza@magisterio.com.co

\section{Juan Carlos Orozco Cruz}

Magister en Docencia de la Física y Licenciado en Física y Matemáticas, Universidad Pedagógica Nacional. Rector, Universidad Pedagógica Nacional. Bogotá, Colombia. orozco@pedagogica.edu.co

\section{Clara Inés Chaparro Susa}

Doctorado en Historia, Lógica y Filosofía de la Ciencia, Universidad Nacional de Educación a Distancia (UNED), Madrid, España. Magister en Docencia de la Física y Licenciada en Física y Matemáticas, Universidad Pedagógica Nacional. Docente de Planta del Departamento de Física de la Universidad Pedagógica Nacional.

chaparro@pedagogica.edu.co

\section{Liliana Lacolla}

Doctora en Enseñanza de las Ciencias, España. Licenciada en Química, Universidad de San Martin. Buenos Aires, Argentina.

lilianaele@yahoo.com.ar

\section{Dino de Jesús Segura Robayo}

Magister en Educación, Universidad de Nueva york, sede Búfalo, Estados unidos. Físico, Universidad de Leipzig, Alemania. Docente de Excelencia. Premio Otorgado por la Alcaldía de Bogotá.

apriori@telecom.com.co

\section{João Batista Siqueira Harres}

Doctor en Educación, Pontificia Universidade Católica do Rio Grande do Sul, Brasil. Maestro en Educación, Pontificia Universidad Católica do Rio Grande do Sul, Brasil. jbharres@univates.br

\section{Roberto Medina Bejarano}

Doctorando en Estudios Sociales, Facultad de Ciencias Sociales y Humanas, Universidad Externado de Colombia, Bogotá. Maestría en Sociedad de la Información y del Conocimiento, Universidad Oberta de Cataluña. Barcelona. Maestría en Literatura. Pontificia Universidad Javeriana, Bogotá. Licenciado en Lingüística y Literatura. Universidad Distrital Francisco José de Caldas.

rmedina@pedagogica.edu.co

\section{Verónica Andrea Catebiel}

Magister en Educación con Énfasis en Enseñanza de las Ciencias, Universidad del Valle. Licenciada en Enseñanza de las Ciencias en Mención en Didáctica de la Química, Universidad Nacional General, San Martin, Argentina, Docente Universidad de la Patagonia Argentina.

veronicacatebiel@gmail.com

\section{Clara Eugenia Amador-Lankster}

Ph.D. University of Southern California, Los Angeles, CA. Ph.D. in Educational Policy and Administration Focus Areas: A Cost-Effectiveness Analysis of Two Multiple Subject (CLAD) Internship Credential Programs in California. National University, Los Angeles, CA. School Of Education. State Coordinator for Teacher Education Internship. Associate Professor, Teacher Education Department. Fulbright Senior Specialist, US Government. amadorwatson@usa.net

\section{Elizabeth B. Ormart}

Doctora en Psicología, Universidad de Buenos Aires. Magister de la Universidad de Buenos Aires en Psicología educacional. Especialidad en Psicología Clínica, GCBA. Licenciada en Psicología, UBA. Profesora en Filosofía y Pedagogía. (Doctorado en curso en Filosofía).

eormart@gmail.com 